Lottermoser u. v.Meyer: Ueber colloïdales Silbers. 241

$\beta$-Benzylhydroxylamin, $p$-toluolsulfinsaures $\beta$-Dibenzylhydroxylamin und Benzylisobenzaldoxim, welches seine Entstehung jedoch nur einer Nebenreaction verdankt. Ein eigenthümliches Verhalten zeigen Acetoxim und $\alpha$-Benzaldoxim, welche beim Verschmelzen mit p-Toluolsulfinsäure, wahrscheinlich unter Bildung eines nicht isolirbaren Zwischenprodukts Aceton, beziehentlich Benzaldehyd, $\mathrm{p}$-toluolsulfonsaures Ammonium und p-Toluolsulfonamid liefern. In vollkommen verschiedener Weise jedoch reagiren Acetoxim und Benzaldoxim mit $\mathrm{p}$-Toluolsulfinsäure in Eisessig; in diesem Falle resultiren bei Anwendung des ersteren als Reactionsprodukte Aceton und p-toluolsulfonsaures Ammonium, Benzaldoxim liefert dagegen ein eigenthümliches Condensationsprodult, welches sich schon durch schwache Alkalien in Benzaldehyd, $p$-Toluolsulfonamid und p-Toluolsulfinsäure zerlegen lässt.

\title{
XXI. Zur Kenntniss des colloïdalen Silbers;
}

Von

A. Lottermoser und E. von Meyer.

(1. Mittheilung.)

So mancherlei Angaben über das Verhalten des colloïdalen Silbers ${ }^{1}$ ) vorliegen, so ist doch nur wenig über die Umwandlung desselben in gewöhnliches Silber, zumal in quantitativer Hinsicht, ermittelt. Anlass zu den im Folgenden mitgetheilten Versuchen gaben Beobachtungen auf medicinischem Gebiete: Hr. Hofrath Dr. Credé (Dresden) verwandte das „lösliche Silber" in Salbenform bei septischen und ähnlichen Erkrankungen mit überraschendem Erfolge. Hieraus konnte

1) Vergl. namentlich Carey Lea, Amer.Journ. science, Bd.37,38, 41. E. A. Schneider, Ber. 24,$3370 ; 25,1440$. Schneider u. Barus, Zeitschr. physik. Chem. 8, 278. Muthmann, Ber. 20, 983.

Journal f. prakt. Chemie [2] Bd, 56. 
242 Lottermoser u. v. Meyer: Ueber collö̈dales Silber.

der Schluss gezogen werden, dass das Silber durch die Haut in das Blut und andere Säfte des Körpers gelangte, mit diesen circulirte und so zur Wirkung kam.

In Widerspruch damit scheint die Thatsache zu stehen, dass colloïdales Silber, in wässriger Lösung ${ }^{1}$ ), durch geringe Mengen Chlornatrium, welches ja in allen Säften des Körpers sich findet, sofort in schwammiges Silber umgewandelt, also ausgeschieden wird.

Eine kurze Ueberlegung und ein daran sich schliessender Versuch führte zur Aufklärung dieses Widerspruches. Fügt man zur Silberlösung Eiweiss (Hühnereiweiss, Blutserum) und dann Kochsalz, so tritt die Fällung des Silbers nicht oder bei Anwendung grösserer Mengen des Salzes nur theilweise ein. Worauf diese Thatsache beruht, vermögen wir noch nicht anzugeben; die Bildung eines Silber-Albuminats erscheint ausgeschlossen. ${ }^{2}$ ) Auch Salzsäure, die das colloïdale Silber sehr leicht fällt, verliert theilweise diese Wirkung, wenn Eiweiss zugegen ist.

Wir stellten uns nun die Aufgabe, das Verhalten von Salzen und Säuren zu collö̈dalem Silber kennen zu lernen. Schon bei der qualitativen Prüfung zeigten sich, je nach der Natur der Säuren und Salze, grosse Unterschiede in der Fähigkeit, das colloïdale Silber zu fällen, so dass es geboten war, die Umwandlung desselben quantitativ zu verfolgen.

Der Uebergang des collö̈dalen in unlösliches Silber, das sich entweder schwammig oder als feine schwarze Fällung abscheidet, lässt sich titrimetrisch unschwer feststellen. Zu diesem Zwecke wird eine wässrige Lösung des collö̈dalen Silbers von bestimmtem Gehalt mit einer ebenfalls bestimmten

1) Dem Sprachgebrauche folgend, benutzen wir hier und weiterhin das Wort „Lösung“ für die Flüssigkeit, welche colloüdes silber scheinbar gelöst enthält, ohne jetzt die Frage zu erörtern, in welchem Zustande man sich dasselbe in dieser tief schwarzhraunen Flüssigkeit zu denken hat.

$\left.{ }^{2}\right)$ Uebrigens wird auch die Ausscheidung von Silber beim Erwärmen einer ammoniakalischen Lösung von milchsaurem oder citronensaurem Silber mit Traubenzucker durch vorherigen Zusatz von Eiw iss verhindert oder stark verzögert; man erhält eine tief rothbraune Lösung. 
Menge Wasser verdünnt, und aus einer Bürette die Lösung der betreftenden Säure, resp. des Salzes (normal, halbnormal etc.) zugetröpfelt. Zuerst beobachtet man nach Zusatz gewisser Mengen einen Umschlag der ursprünglich kaffeebraunen Farbe in Grün; dieser Punkt ist ziemlich scharf bestimmbar. Dann erfolgt, nach weiterem Zusatz Ausscheidung des Silbers, deren Beendigung man durch eine Tüpfelprobe annähernd genau beobachten kann: sobald nämlich ein Tropfen der Flüssigkeit, auf Filtrirpapier gebracht, farblos ausfliesst und man die feinen Silbertheilchen scharf sieht, ist der Endpunkt erreicht.

Die folgenden Versuche sind mit einer 0,5 procent. Lösung des colloïdalen Silbers ausgeführt; es sei aber betont, dass zu dieser ersten Reihe von Versuchen ein colloïdales Silber ${ }^{1}$ ) angewandt wurde, das nicht völlig - - in Folge der Gewinnungsweise - von citronensaurem Ammon befreit war; das dem festen Präparat beigemengte unlösliche Silber kam nicht in Betracht, da es durch Decantiren beseitigt wurde.

\section{Versuche mit Säuren.}

Die Säuren fällen collö̈dales Silber als feinzertheiltes "molekulares" (Salpetersäure löst das zuerst ausgeschiedene $\mathrm{Ag}$ beim Erwärmen wieder auf). Die Beobachtungen deuten auf eine Beziehung zwischen Fällungsvermögen und Affinitätsgrösse der Säuren. Auch die Verdünnung und die Zeit, welche die Umwandlung braucht, sind von sehr grossem Einfluss.

$\mathrm{Zu}$ den Versuchen wurden stets die gleichen Mengen Silberlösung: $5 \mathrm{Ccm}$. (mit 0,025 Grm. Ag) angewandt. In der folgenden Tabelle enthält die I. Columne die Säure und ihre Concentration. Die Verdünnung der Silberlösung und ihr Procentgehalt sind in der II. Columne angegeben; in der III. und IV. Columne findet sich der Verbrauch in Cubikcentimetern der betreffenden Säuren zur Erreichung des Um. schlages in Grün und zur Ausfällung aufgeführt.

1) Wir verdanken das werthvolle Präparat dem Entgegenkommen der chemischen Fabrik von Heyden in Radebeul bei Dresden. 
244 Lottermoser u. v.Meyer: Ueber colloïdales Silber.

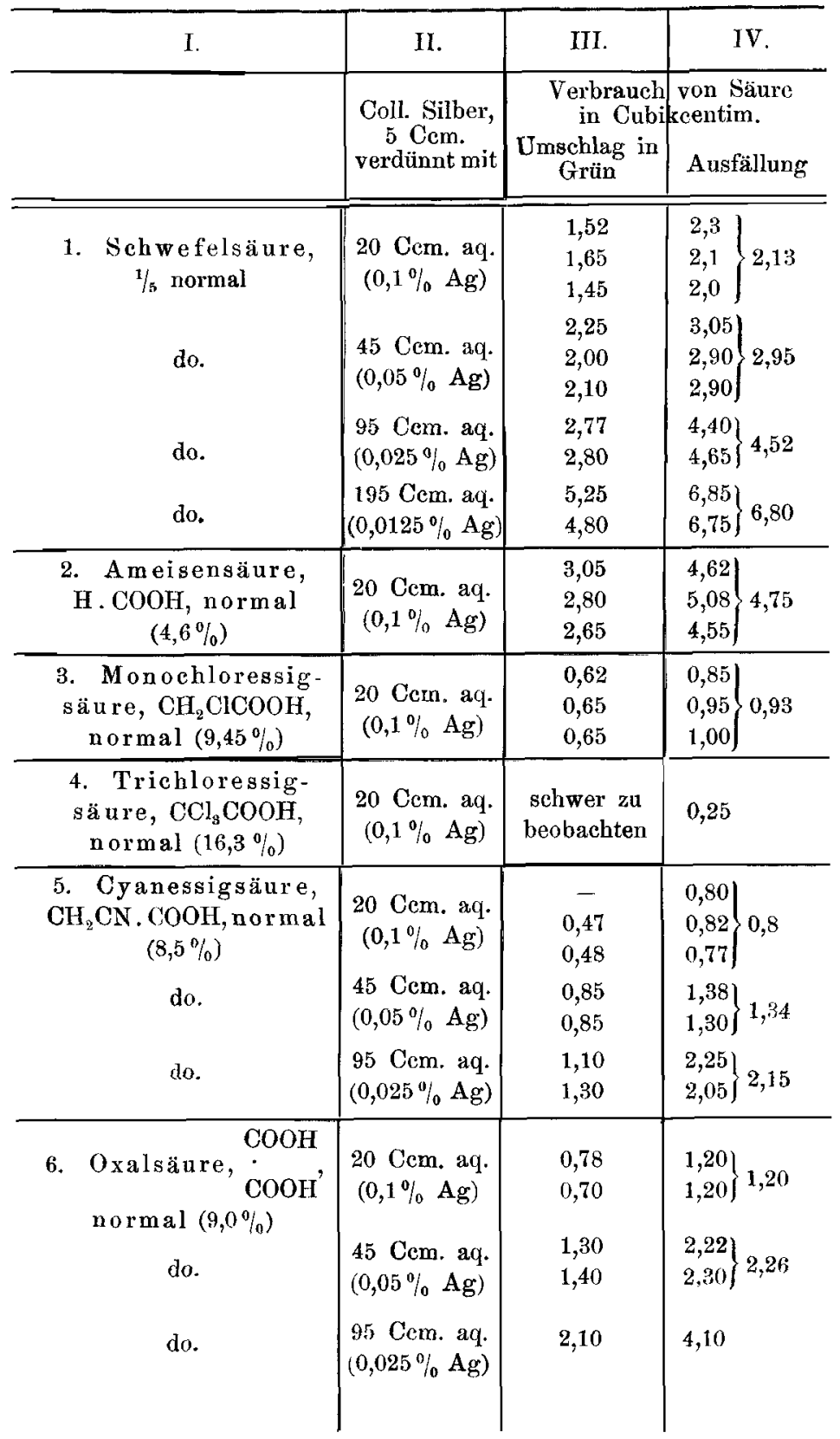


Lottermoser u. v. Meyer: Ueber colloïdales Silber. 245

\begin{tabular}{|c|c|c|c|}
\hline I. & II. & III. & IV. \\
\hline & $\begin{array}{l}\text { Coll. Silber, } \\
5 \text { Ccm. } \\
\text { verdünnt mit }\end{array}$ & $\begin{array}{r}\text { Verbrauch } \\
\text { in Cubi } \\
\text { Umsehlag in } \\
\text { Grün }\end{array}$ & $\begin{array}{l}\text { von Säure } \\
\text { ceutim. } \\
\text { Ausfällung }\end{array}$ \\
\hline $\begin{array}{c}\text { 7. Malonsäure, } \\
\mathrm{CH}_{2} \cdot(\text { COOH })_{2} \text {, normal } \\
(10,4 \%) \\
\text { do. } \\
\text { do. }\end{array}$ & 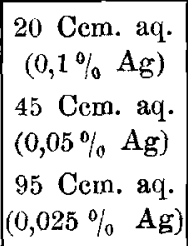 & $\begin{array}{l}1,48 \\
1,50 \\
2,28 \\
2,28 \\
3,25 \\
3,07\end{array}$ & 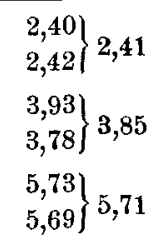 \\
\hline $\begin{array}{c}\text { 8. Bernsteinsäure, } \\
\mathrm{C}_{2} \mathrm{H}_{4}(\mathrm{COOH})_{2} \text {, normal } \\
(11,8 \%)\end{array}$ & $\begin{array}{l}20 \text { Cem. aq. } \\
(0,1 \% \mathrm{Ag})\end{array}$ & $\begin{array}{r}9,40 \\
10,10\end{array}$ & $\left.\begin{array}{l}16,10 \\
16,40\end{array}\right\} 16,25$ \\
\hline $\begin{array}{l}\text { 9. W einsäure, } \\
. \mathrm{C}_{2} \mathrm{H}_{2}(\mathrm{OH})_{2}(\mathrm{COOH})_{3}, \\
\text { normal }(15 \%)\end{array}$ & $\begin{array}{c}20 \text { Cem. aq. } \\
(0,1 \% \text { Ag) }\end{array}$ & $\begin{array}{l}2,90 \\
2,50\end{array}$ & $\left.\begin{array}{l}3,70 \\
3,80\end{array}\right\} 3,75$ \\
\hline
\end{tabular}

Mustert man die unter möglichst gleichen Bedingungen, mit Silberlösungen gleicher Concentration $(0,1 \%)$ ausgeführten Versuche, so ist eine Beziehung der zur Ausfällung des Silbers nöthigen Mengen von Säuren zu deren Affinität bemerkbar. Je grösser diese, desto geringere Mengen der Säure sind zur Umwandlung in unlösliches Silber erforderlich. Streng gilt diese umgekehrte Proportionalität zwar nicht, doch ist sie unverkennbar. Man vergleiche z. B. die gefundenen Mengen Säure (in Cubikcentim.) mit den Zahlen, die Ostwald für die Leitfähigkeit derselben (in normaler Lösung) angegeben hat (auf 100 als Werth der Salzsäure bezogen).

\begin{tabular}{|c|c|c|}
\hline & $\begin{array}{l}\text { Leitfähig- } \\
\text { keit: }\end{array}$ & $\begin{array}{l}\text { Ausfällende } \\
\text { Menge: }\end{array}$ \\
\hline Ameisensäure & 1,68 & 4,75 \\
\hline Chloressigsäure & 4,90 & 0,93 \\
\hline Trichloresssigsäur & 62,3 & 0,25 \\
\hline Oxalsäure. & 19,7 & 1,2 \\
\hline Malonsäure. & 3,1 & 2,4 \\
\hline Bernsteinsäure. & 0,58 & 16,25 \\
\hline Weinsäure. & 2,28 & 3,75 \\
\hline
\end{tabular}

Die Cyanessigsäure ist nach Ostwald's Messungen wie auch die obigen Werthe zeigen - merklich stärker, als die Chloressigsäure. 
246 Lottermoser u. v.Meyer: Ueber collö̈dales Silber

Dass von der Verdünnung die Menge der zur Umwand lung des collöidalen Silbers nöthigen Säure abhängig ist, erkennt man aus den Versuchen 1, 5, 6, 7. Wenn man in Rücksicht zieht, dass die Zeit grossen Einfluss auf diese Umwandlung ausübt, so kann man wohl an ein bestimmtes Verhältniss zwischen den Säuremengen und der Verdünnung denken. Wir haben beobachtet, dass jene Umwandlung schon mit Hilfe viel geringerer Mengen derselben Säure eintritt, wenn man nur diese längere Zeit auf die Silberlösung wirken lässt. Um den Einfluss dieses Factors näher za bestimmen, sollen weitere Versuche angestellt werden.

Einwirkung von Salzen auf colloidales Silber.

Die Wirkung der Salze auf die Silberlösung äussert sich sehr verschiedenartig je nach der Natur der Salze: die Alkaliund Ammoniumverbindungen der schwer lösliche Silbersalze bildenden Säuren, also der Halogen - und Rhodan-wasserstoffsäuren, der Chrom- und Kohlensäure fällen das Silber als unlösliches, und dazu sind geringe Mengen der Salze erforderlich (z. B. bei Anwendung von $25 \mathrm{Ccm}$. einer etwa 0,1procent. Lösung colloïdalen Silbers $0,6 \mathrm{Ccm}$. einer normalen Kaliumchromat - und ebenso viel einer halbnormalen Kaliumdichromat-Lösung). Die Versuche sollen erst wiederholt und ergänzt werden, ehe wir ihre Einzelnheiten mittheilen.

Die Salze der Schwermetalle (auch Erdmetalle) bewirken mit noch geringeren Mengen die obige Umwandlung. - Lösungen von Eisen- oder Quecksilberchlorid führen das collö̈dale Silber in Chlorsilber über und enthalten sodann die Chlorüre.

Die Alkali- und Ammoniumsalze von Säuren, deren Silbersalze löslich sind, verhalten sich wiederum ganz anders: sie bewirken nur eine partielle Ausfällung unlöslichen Silbers, scheiden aber einen beträchtlichen Theil des colloïdalen Silbers als "„ösliches" ab. Man hat es also mit einer Aussalzung zu thun. Die zu solcher Ausscheidung des Silbers erforderlichen Mengen der Salze sind erheblich grösser, als die in obigen Fällen gebrauchten und sind, unter gleichen Bedingungen, einander gleich (auf je $25 \mathrm{Ccm}$. einer 0,1 procent. Silberlösung wurden verbraucht je $14 \mathrm{Ccm}$. der Normallösungen von $\mathrm{Na}_{2} \mathrm{SO}_{4}$, $\left.\mathrm{H}_{4} \mathrm{~N}\right)_{2} \mathrm{SO}_{4}, \mathrm{KNO}_{3}, \mathrm{NaO}\left(\mathrm{COCH}_{3}\right)$ ). 


\section{Collo ïdales Silber und Halogene.}

Halogene verwandeln das colloïdale Silber leicht in die entsprechenden Salze, die das Verhalten collö̈daler Körper zeigen. Am besten lässt sich dies beim Jodsilber beobachten. Fügt man zur verdünnten Lösung des collö̈dalen Silbers eine alkoholische Jodlösung von bestimmtem Gehalt $(3 \%)$, so tritt der Endpunkt der Reaction durch Farbenumschlag scharf ein; jedoch beobachtet man - in Folge der Bildung von Jodoform (aus dem beigemengten Ammoniumcitrat und Alkohol) - einen zu grossen Verbrauch von Jod. Die Versuche sollen mit möglichst reinem colloïdalen Silber wiederholt werden. Die dabei entstandene Lösung ist milchig, gelblich-weiss, bei genügender Verdünnung durchsichtig, hält sich längere Zeit unverändert, wird aber durch geringe Mengen Salpetersäure und anderer Mineralsäuren sofort gefällt, unter Abscheidung des gesammten Jodsilbers. Vielleicht ist es möglich, durch feine Messungen den Betrag der Wärmetönung festzustellen, die beim Uebergange des colloïdalen in das unlösliche Jodsilber stattfindet.

Schliesslich sei ein Versuch kurz erwähnt, der die Wirkung des elektrischen Stromes auf das colloïdale Silber zum Gegenstand hatte. Wie schon Barus und Schneider beobachteten, ist die schwache Leitung, die dasselbe in wässriger Lösung zeigt, den Begleitsubstanzen zuzuschreiben. Die Nichtleitung des Stroms durch das colloïdale Silber gilt als Stütze der Annahme, dass es sich um eine Suspension feinster Theilchen handele. - So zeigte sich auch bei unserem Versuche sehr schwache Leitung des Stromes, der jedoch eine Wanderung von Silbertheilchen bewirkte: an der Kathode fand sich nach einiger Zeit graues, schwammiges Silber, an der Anode ein brauner Schlamm abgeschieden, der sich in Wasser mit grüner Farbe löste und nur Silber enthielt. Beim Trocknen im Exsiccator ging diese Modification in eine goldgelbe über, die in Wasser unlöslich war.

Die obigen Versuche, so lückenhaft sie noch sind, zeigen deutlich, dass man es mit eigenartigen Verhältnissen zu thun hat, deren nähere Erforschung unsere Kenntnisse von colloïdalen Körpern sicherlich erweitern und vertiefen wird.

Dresden, August 1897. 